\title{
QTL analysis of seed dormancy in Arabidopsis using recombinant inbred lines and MOM mapping
}

\author{
WYBE VAN DER SCHAAR§†, CARLOS ALONSO-BLANCO†, KAREN M. LÉON- \\ KLOOSTERZIEL $\uparrow$, RITSERT C. JANSEN + , JOHAN W. VAN OOIJEN $\$$ \\ \& MAARTEN KOORNNEEF*† \\ $\dagger$ Department of Genetics, Wageningen Agricultural University, Dreijenlaan 2, 6703 HA, Wageningen and $\ddagger$ CPRO- \\ DLO, PO Box 16, 6700 AA, Wageningen, The Netherlands
}

\begin{abstract}
The genetic differences for seed germination between two commonly used Arabidopsis thaliana ecotypes Ler and Col, both showing a low level of seed dormancy, were investigated. The analysis was performed with 98 recombinant inbred lines (RILs) derived from the cross between the two ecotypes, and these lines had previously been analysed for molecular marker composition by Lister and Dean (Norwich, UK). The analysis of germination was performed on seeds grown in three different maternal environments and each seed batch was tested in three different germination environments: in light, in darkness and in the presence of the gibberellin inhibitor paclobutrazol. Fourteen loci were identified using the multiple-QTL-model $(\mathrm{MQM})$ procedure for mapping quantitative trait loci. At nine loci no significant interaction between the detection of the locus and environmental factors could be detected. However, three other distinct loci controlling the germination behaviour in the presence of the gibberellin inhibitor paclobutrazol had a much lower or no effect when germination was tested in water either in light or darkness. Two other loci affecting germination in darkness and/or light had practically no effect on germination in the presence of paclobutrazol.
\end{abstract}

Keywords: Arabidopsis, gibberellin inhibitors, QTL mapping, recombinant inbred lines, seed dormancy.

\section{Introduction}

Many traits that differ genetically within and between natural populations of a species can be considered as quantitative traits. Both their multigenic inheritance and the strong effect of environmental factors on such traits make them difficult to analyse in terms of effects of allelic variation at specific loci. Of the traits with large genetic variation in nature, seed dormancy is probably one of the most complicated. Dormancy is defined as the temporary failure of a viable seed to germinate in conditions that favour germination when the restrictive state has come to an end' (Simpson, 1990). For cultivated plants a high level of dormancy is not desirable because it can result in low germination. However, the complete absence of dormancy may result in premature sprouting on the plant, which is

*Correspondence. E-mail: maarten.koornneef@botgen.el.wau.nl §Present address: PBI Cambridge, Maris Lane, Trumpington, Cambridge, CB2 2LQ, U.K. a detrimental property. For wild plants, the degree of seed dormancy is an important factor, ensuring germination in an ecologically favourable period. Genetic differences in seed dormancy within and between 'wild' populations have been observed by many authors (Garbutt \& Witcombe, 1986; Platenkamp \& Shaw, 1993 and references therein). In such genetic studies, the inheritance of dormancy is often described in terms of quantitative inheritance (Lane \& Lawrence, 1995) and in several cases a maternal component could be identified (Platenkamp \& Shaw, 1993). The latter is not unexpected because genetically different tissues (testa, endosperm and embryo) together determine the germination behaviour and thereby the dormancy of seeds. Only in a few cases, e.g. for the maternal component of seed dormancy in Sinapis arvensis, has a single locus been identified (Garbutt \& Witcombe, 1986).

The dissection of complicated genetic traits into their genetic components has been made possible by the use of molecular marker loci and by the 
development of mathematical methods to analyse the genetic relationships between the markers and the quantitative trait. These new methods are much more efficient than the methods of classical quantitative genetics and allow the mapping and study of individual quantitative trait loci (QTLs) (Jansen, 1996). QTLs can be analysed in almost any type of segregating population. However, recombinant inbred lines (RILs: Burr \& Burr, 1991) offer specific advantages because of the ability to repeat an experiment almost indefinitely and in different environments. This is especially important in those situations where environmental effects are large and a strong genotype-by-environment interaction is present. In addition, the lack of heterozygotes simplifics genetic segregation per locus because only two, instead of three, genotype classes exist. However, a drawback of the lack of heterozygotes is that dominance effects cannot be estimated. The effectiveness of a QTL approach for the analysis of seed dormancy by using an immortal mapping population was demonstrated for the first time in wheat by Anderson et al. (1993) and in barley by Ullrich et al. (1993).

In Arabidopsis thaliana natural ecotypes show considcrable genetic variation for germination behaviour (Laibach, 1951; Evans \& Ratcliffe, 1972; Ratcliffe, 1976). The suitability of Arabidopsis for molecular genetic studies and its acceptance as a model plant (Meyerowitz, 1987) will allow the establishment, in the near future, of the relationship between accurately mapped traits and molecular information. Nevertheless, the great variability in seed material coming from the same homozygous genotype and the relatively rapid changes during dry storage in Arabidopsis, led Derkx \& Karssen (1993) to the conclusion that seed germination is a very difficult trait to analyse in this species. For this reason, in order to detect genetic differences in degree of dormancy with sufficient accuracy, the environmental factors acting during the development of dormancy on the mother plant, the storage conditions of the seeds and the germination tests have to be kept identical. Two reports have been published on the genetic analysis of differences in light dependency of different ecotypes. Kugler (1951) showed that the light dependency of germination of the ecotype Hannovrisch Münden ( $\mathrm{Hm}$ ) was recessive in crosses with the dark germinating ecotypes Stockholm (St) and Haarlem (Haa). The comparison of reciprocal crosses indicated that this property was controlled by the genotype of the embryo. NappZinn (1975) analysed $F_{3}$ lines derived from the $\mathrm{Hm} \times \mathrm{St}$ cross and suggested that three genes controlled the difference in light requirement between both parents and that this trait might be linked to flowering time genes segregating in the same population.

In the present study we have analysed the genetic differences between two frequently used pure lines (ecotypes) of $A$. thaliana, viz. Landsberg erecta (Ler) and Columbia (Col), which show rclatively small differences in germination behaviour. For this, we used a set of RILs developed from a cross of Ler and $\mathrm{Col}$ and analysed for marker genotype by Lister \& Dean (1993). It is known that results under different germination test conditions often correlate if the same seed batch is used (Derkx \& Karssen, 1993). However, exceptions have been reported and may indicate that germination promoting factors such as light, nitrate and gibberellins are not always acting in the same way and therefore may sometimes act on different mechanisms with a different genetic basis. The possibility of testing 'identical' genetic material in different maternal environments and different germination test conditions (germination environments) allowed the genetic dissection of this complex trait by analysing the interaction of specific loci with these two types of environments. The present approach was previously used to analyse the genetics of flowering time by using the same genetic material and by applying similar statistical methods (Jansen et al., 1995).

\section{Material and methods}

\section{Plant material}

A set of $98 \mathrm{~F}_{8}$ recombinant inbred lines (RILs) derived from a cross between the $A$. thaliana ecotypes Landsberg erecta (Ler) and Columbia (Col) was analysed for seed germination characteristics. These RILs have been developed by Lister \& Dean (1993) and were provided by the Nottingham Arabidopsis Stock Centre (NASC). The parental genotypes were also included in the experiments. A data set with the genotypes of these RILs for 186 molecular markers and the morphological marker erecta was provided by Lister and Dean. These markers cover $601 \mathrm{cM}$, which is more than 95 per cent of the Arabidopsis genome with an average genetic distance of $4 \mathrm{cM}$, and the largest distance between two markers was $15 \mathrm{cM}$. The parental $L E R$ wild type of the Ler laboratory ecotype (Rédei, 1962) was obtained from Dr Rédei and shown by AFLP analysis to be isogenic (C. Alonso-Blanco \& A. J. M. Pceters, unpublished data). 


\section{Maternal environments}

To produce seeds of each RIL and the two parents under identical conditions, seeds of each genotype were sown on water-soaked filter paper in $6 \mathrm{~cm}$ plastic Petri dishes and incubated for 3 days in a cold room $\left(4^{\circ} \mathrm{C}\right)$ to overcome remaining dormancy. Thereafter the seeds were incubated for 2 days in a climate room $\left(25^{\circ} \mathrm{C}, 16 \mathrm{~h} \mathrm{light/day)}\right.$ and the germinated seeds were planted in a greenhouse. Each seed was carefully taken with a small brush and put on a clay pot filled with potting compost. The seed production consisted of four plants per RIL and 12 plants for each of the parental genotypes, which were grown in a completely randomized design. All the genotypes were from the same original seed lot provided by the NASC stock centre and were grown at three different periods throughout the year in the same air-conditioned greenhouse with supplementary light provided in winter time, maintaining long day conditions (from 21 September to 21 March). Dates for sowing, harvesting and germination testing are listed in Table 1. The three periods provide three different maternal environments during seed development and seed maturation with differences in light intensity, light quality, temperature and humidity. For each maternal environment the fully mature seeds were harvested on the same day from each individual plant and stored in cellophane bags at room temperature.

\section{Germination environments}

All RIL genotypes from each of the three maternal environments were analysed in three different germination environments resulting in a total of nine different randomized experiments: germination in light, in darkness and in paclobutrazol. To each experiment the parents were added but they were not randomized. The germination tests were performed in $6 \mathrm{~cm}$ plastic Petri dishes with filter paper soaked with $1.5 \mathrm{~mL}$ demineralized water (Léon-Kloosterziel et al., 1996a). Approximately 50 seeds harvested from each single plant were evenly distributed in a separate Petri dish. For light germination tests, performed with seeds stored for only 2 weeks, the dishes were put in plastic trays with filter paper saturated with tap water, covered with a transparent lid and kept at $25^{\circ} \mathrm{C}$ in a climate chamber with $38 \mathrm{~W}$ Philips TL84 fluorescent tubes at $\approx 8 \mathrm{~W}$ $\mathrm{m}^{-2}$ for $16 \mathrm{~h}$ followed by $8 \mathrm{~h}$ darkness. For dark germination experiments, performed 4-5 weeks after harvesting, the individual Petri dishes were wrapped in aluminium foil and the tray in which they were incubated was covered with two black polythene bags. Five to eight weeks (Table 1) after harvest, seeds were sown in $10 \mu \mathrm{M}$ paclobutrazol (ICI, Bracknell, UK), which is an inhibitor of gibberellin biosynthesis. Gibberellins are required for the germination of dormant seeds (Koornneef \& Karssen, 1994). Subsequently these seeds in paclobutrazol were incubated at $4^{\circ} \mathrm{C}$ for three days before they were transferred to $25^{\circ} \mathrm{C}$ in light as described above for the light germination test. In all tests the total number and the number of germinated seeds were counted after incubation for 1 week at $25^{\circ} \mathrm{C}$. To analyse the changes of the parents in light germination, seeds from the same harvest were tested as described above at weekly intervals until 100 per cent of the seed batch germinated. From these experiments, the after-ripening requirement was estimated as days of dry storage required to reach 50 per cent germination.

\section{Statistical and QTL analysis}

For each plant the percentage of germinated seeds was obtained. Four plants per RIL were tested and the average germination percentage was determined and transformed by the angular $(=\arcsin \sqrt{ })$ transformation to improve the normality of the data. The angular transformed line means were used to estimate correlation coefficients and to perform the QTL analysis. This analysis was performed by applying multiple-QTL-model (MQM) mapping, an approach that can accommodate the mapping of multiple QTLs and QTL-environment interactions. The method consists of two stages. In the first stage,

Table 1 Dates on which Arabidopsis plants were handled and when seeds were incubated for germination tests

\begin{tabular}{llllll}
\hline $\begin{array}{l}\text { Maternal } \\
\text { environment }\end{array}$ & $\begin{array}{c}\text { Planting } \\
\text { date }\end{array}$ & $\begin{array}{c}\text { Harvest } \\
\text { date }\end{array}$ & $\begin{array}{c}\text { Germination } \\
\text { in light }\end{array}$ & $\begin{array}{c}\text { Germination } \\
\text { in darkness }\end{array}$ & $\begin{array}{c}\text { Germination } \\
\text { in paclobutrazol }\end{array}$ \\
\hline 1 & $13 / 7 / 94$ & $26 / 8 / 94$ & $7 / 9 / 94$ & $27 / 9 / 94$ & $20 / 10 / 94$ \\
2 & $14 / 9 / 94$ & $2 / 11 / 94$ & $16 / 11 / 94$ & $5 / 12 / 94$ & $29 / 12 / 94$ \\
3 & $1 / 12 / 94$ & $1 / 2 / 95$ & $16 / 2 / 95$ & $1 / 3 / 95$ & $10 / 3 / 95$ \\
\hline
\end{tabular}


the trait is regressed jointly on the nine environments, on multiple markers (36) and on the interactions between markers and environments. We used a separate residual variance parameter for each environment. The 36 markers were chosen from the 187 available markers to provide uniform coverage of the genetic map. A backward elimination procedure was then used to identify the important markers and the important marker-environment interactions. Redundant markers and interactions were dropped on the basis of a 2 per cent significance level per test. A marker could only be dropped after an earlier removal of its interaction. In the second stage of MQM mapping, the selected markers and interactions were used as genetic background control in precision mapping (as in interval mapping) of QTLs, and QTL likelihood maps were produced. While calculating the QTL likelihood at a certain map position, only selected markers and interactions outside a 'window' around the location of current interest were used as cofactors in the regression model; window size was initially set to $10 \mathrm{cM}$, but was changed interactively on the basis of the curvature of QTL likelihood in some cases (smaller window size in the case of high QTL likelihood, broader in the case of low QTL likelihood). Finally, four markers were added to the set of 36 markers in order to assess the QTL likelihood more accurately in regions displaying considerable QTL activity. The genome-wide significance thresholds for QTL and QTL-environment interaction were obtained by computer simulation, using the same observed marker data but new simulated trait values. More details about MQM mapping, such as how to deal with missing marker observations, can be found in Jansen (1994) and Jansen \& Stam (1994).

\section{Results \\ Variability in seed germination of the parents}

The two parental and genetically homozygous ecotypes Ler and Col are the most commonly used laboratory genotypes in Arabidopsis. Major differences in germination behaviour have not been reported for these ecotypes. However, when the germination of seed batches of both genotypes grown and tested under identical conditions were compared, differences were observed. As is shown in Fig. 1, the difference for after-ripening requirement in light between seeds harvested from plants growing in different conditions, was often larger than the genetic differences. Although in most experiments Col seeds had a lower after-ripening requirement, and therefore were less dormant, the differences between the two ecotypes depended on the seed harvest period. In the experiments presented in Fig. 1 the germination percentage 1 week after harvest correlated well with the after-ripening requirement (Fig. 2). Therefore, a single germination test will characterize to a large extent the germination behaviour of a genotype harvested in a specific maternal environment. In most experiments the germination of Ler seeds under light was lower than that of Col seeds, indicating that $\mathrm{Col}$ were slightly less dormant. In addition, when seeds were germinated in $10 \mu \mathrm{M}$ paclobutrazol, which inhibits gibberellin (GA) biosynthesis, Col was more resistant than Ler (Fig. 3). This positive correlation between germination of freshly harvested seeds and germination in the presence of GA inhibitors has been found for several nondormant mutants in Arabidopsis (Léon-Kloosterziel et al., 1996a). Variation for the level of resistance to these inhibitors
Fig. 1 Number of days of dry storage until 50 per cent of the seeds of the ecotypes Ler (black bars) and Col (light grey bars) germinated in a light germination experiment after a period of dry storage. The experiment number indicates month and year that the seeds were harvested.

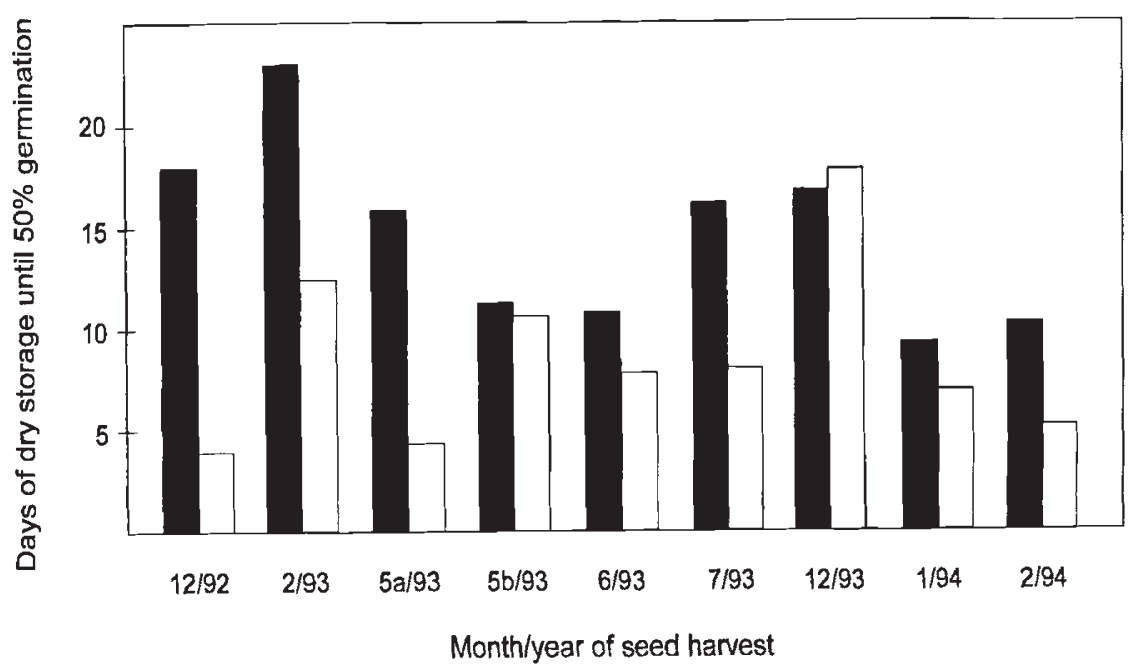




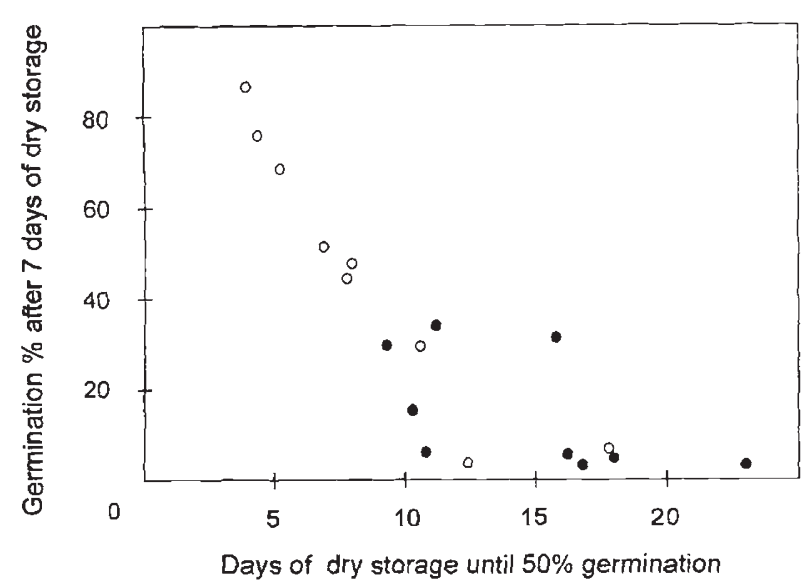

Fig. 2 Relationship between number of days of storage to reach 50 per cent germination (in light) and the germination percentage 1 week after harvest for the Arabidopsis ecotypes Ler (closed symbols) and Col (open symbols) harvested at different times, as indicated in Fig. 1.

between seeds grown in different maternal environments has been reported before (Léon-Kloosterziel et al., 1996a). In contrast, the germination in darkness of Ler seeds was generally higher than that of Col seeds (Fig. 4), although again variation among seeds grown in different maternal environments was observed.

\section{Segregation among the R/Ls}

The frequency distribution of the germination percentages per RIL and the range for the parental ecotypes are shown in Fig. 4. As was observed in the above-described experiments, the differences between the parents, when comparing maternal environments, were variable for all three germina-

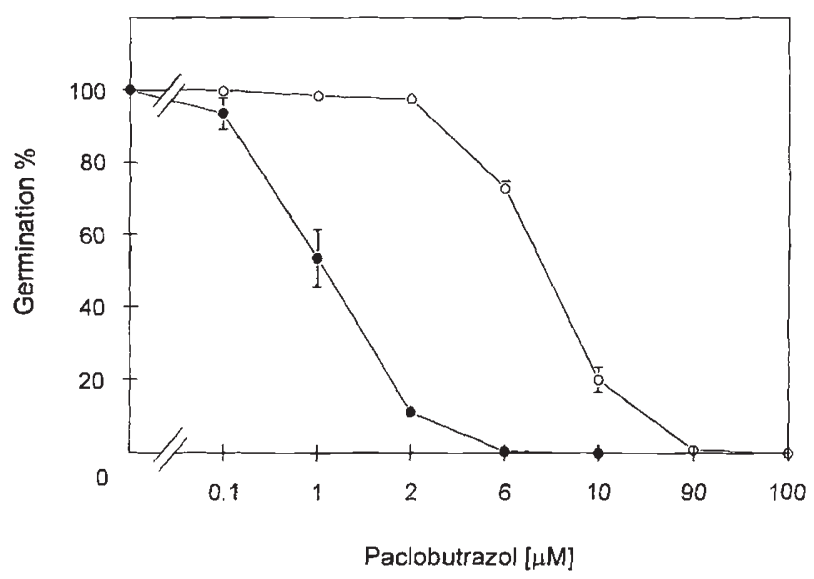

Fig. 3 Paclobutrazol sensitivity for germination of the Ler and Col ecotypes of Arabidopsis. tion environments. The frequency distribution of the RILs for germination percentage did not allow the detection of simple segregation ratios. The germination range of the RILs exceeded the values for the parental genotypes, indicating that both parents have positively and negatively acting alleles at different loci. The correlation matrix among the average values per RIL in the different experiments is shown in Table 2. On the one hand, the correlation coefficients between pairs of experiments of the same germination environment are highly significant despite the quantitative values being very different. On the other hand, when comparing different germination environments, either from the same or from different maternal environments, the data often do not correlate. This is particularly the case when germination tests in paclobutrazol are compared with germination in light and in darkness. This lack of correlation suggests that germination in the three different germination environments does not reflect traits that are genetically completely identical.

\section{The map location of seed dormancy genes}

In the MQM approach, the data from all three maternal and three germination environments were analysed simultaneously. The fractions of phenotypic variances explained by 36 markers, uniformly distributed over the genetic map, are shown in Table 3 for each of the nine experiments. The genetic differences between the two parents reside at 14 different positions on the Arabidopsis genetic map (Fig. 5). The relative allele effect at each locus was rather small, being $15 \arcsin \sqrt{ }$ units for the two larger effect QTLs on chromosome 2. Nevertheless, map positions of the different QTLs could be detected more accurately than with QTL mapping procedures such as single marker or interval mapping. This was particularly true where linked QTLs with opposite effects were present, on chromosomes 2,3 and 5. Ler alleles at seven loci promote germination as compared with the Col alleles, whereas at seven loci the Ler alleles have a negative effect. The presence of both germination-promoting and germinationreducing alleles in both parents is in agreement with the transgression observed in many RILs. At nine of these QTLs no significant interaction with either the maternal or germination environment could be detected. However, significant interactions were observed at the other five loci. On chromosome 2 a strong effect on dark germination was detected around the ER locus, whereas this QTL has a smaller effect on germination in light and no effect on germination in paclobutrazol. However, for the 
neighbouring locus (near m336) a paclobutrazol germination locus with a strong effect is detected, whereas in dark germination it showed no effect. On chromosome 3 , a somewhat similar complex situation could be observed. Data for germination in light indicated a QTL which is not found in paclobutrazol, whereas at the neighbouring locus (between $\mathrm{m} 457$ and $\mathrm{m} 424$ ) the opposite was observed. A third paclobutrazol-specific locus was found on chromosome 5 near w69. For all these paclobutrazol-specific loci, a light germination effect is observed, although the effect on germination in paclobutrazol is larger. Ler alleles at these loci have a negative effect, which is in agreement with the relative sensitivity of Ler to this inhibitor of germination. The interaction between the detected QTLs and maternal environment was limited. Only on chromosome 3 were QTLs detected in maternal environments one and two, but these were not found in the third environment which is characterized by almost no germination in darkness and paclobutrazol (Fig. 4). This combined analysis of the three germination environments clearly shows that some of the loci control germination/dormancy in a general way, whereas other loci affect aspects of germination in specific conditions.

On chromosome 2 a QTL was detected around the $E R$ locus. This marker was included in the analy-

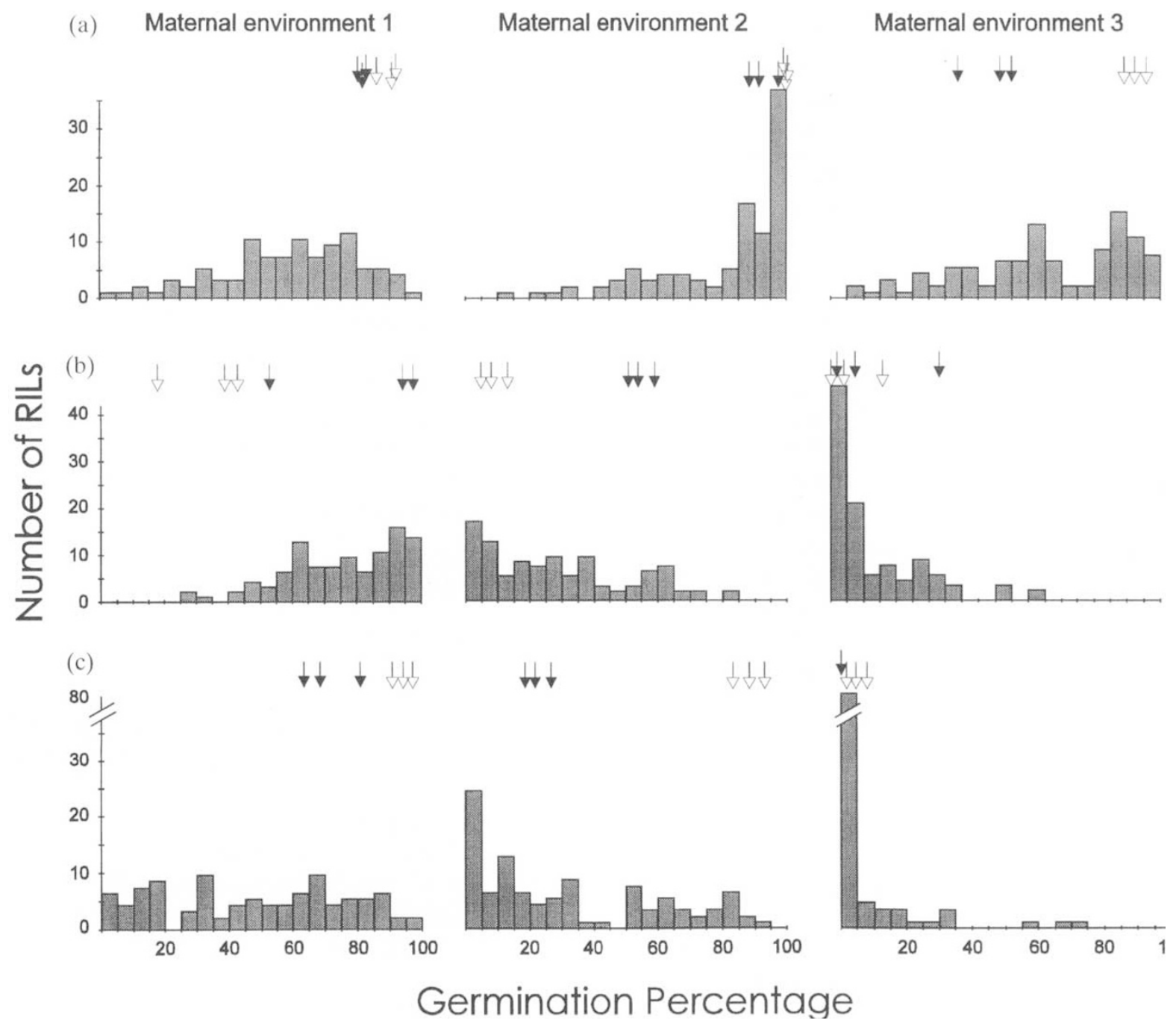

Fig. 4 Frequency distributions of germination percentages of 98 RILs of Arabidopsis based on the average germination of seed samples of four plants per line: (a) germination in light; (b) germination in darkness; and (c) germination in $10 \mu \mathrm{M}$ paclobutrazol. Black arrows indicate averages of germinations tests of four plants of the Ler parent, and white arrows average percentages of four plants of Col to allow comparison with the four replicates per RIL. In total, 12 plants (three groups of four) of each Ler and Col were included in the analysis. 
Table 2 Correlation coefficients between germination percentages of Arabidopsis obtained in three different germination environments within and between three maternal environments, based on angular transformed averages of 98 RILs

\begin{tabular}{|c|c|c|c|c|c|c|c|c|}
\hline & \multicolumn{8}{|c|}{ Germination in: } \\
\hline & \multicolumn{3}{|c|}{ light } & \multicolumn{3}{|c|}{ darkness } & \multicolumn{2}{|c|}{ paclobutrazol } \\
\hline & $\mathrm{ME} \dagger 1$ & ME 2 & ME 3 & ME 1 & ME 2 & ME 3 & ME 1 & ME 2 \\
\hline \multicolumn{9}{|l|}{ Light } \\
\hline ME 2 & $0.39^{* *}$ & & & & & & & \\
\hline ME 3 & $0.47^{* *}$ & $0.70^{* *}$ & & & & & & \\
\hline \multicolumn{9}{|l|}{ Darkness } \\
\hline ME 1 & $0.28^{* *}$ & 0.00 & 0.17 & & & & & \\
\hline ME 2 & $0.24^{*}$ & $0.36^{* *}$ & $0.30^{* *}$ & $0.25^{* * *}$ & & & & \\
\hline ME 3 & $0.20^{*}$ & $0.34^{* *}$ & $0.45^{* *}$ & $0.27 * *$ & $0.54 * *$ & & & \\
\hline \multicolumn{9}{|l|}{ Paclo $\neq$} \\
\hline ME 1 & 0.08 & 0.07 & $0.22^{*}$ & -0.13 & 0.17 & 0.14 & & \\
\hline ME 2 & $0.20^{*}$ & $0.49^{* *}$ & $0.40^{* *}$ & -0.11 & $0.35^{* *}$ & $0.33^{* *}$ & $0.44^{* *}$ & \\
\hline ME 3 & 0.17 & 0.13 & $0.34^{\text {*** }}$ & 0.10 & -0.08 & 0.05 & $0.24 * *$ & $0.40^{\text {*** }}$ \\
\hline
\end{tabular}

$† \mathrm{ME}$, maternal environment.

$\ddagger$ Paclo, germination in light in $10 \mu \mathrm{M}$ paclobutrazol.

${ }^{*} P<0.05 ;{ }^{*} P<0.01$.

sis because the recessive allele, which was induced by X-ray mutagenesis, was present in Ler (Rédei, 1962) and therefore segregates in the RIL population. This gene controls the elongation of stems, petioles and also some aspects of leaf and silique morphology (Rédei, 1962). It cannot be excluded that this locus may have an effect on seed germination, because this process has a cell elongation component when the radicle protrudes through the seed coat. Therefore, the LER genotype, differing from Ler only at the er locus, was compared with its isogenic counterpart. The germination of seeds from 10 plants of the genotypes Ler, LER and Col, grown in the same maternal environment, was tested under the same conditions as used for the RIL experi-

Table 3 Fraction of phenotypic variance explained by multiple regression on 36 markers equally distributed over the Arabidopsis genome

\begin{tabular}{lccc}
\hline \multirow{2}{*}{$\begin{array}{l}\text { Maternal } \\
\text { environment }\end{array}$} & light & darkness & paclobutrazol \\
\cline { 2 - 4 } 1 & 0.08 & 0.28 & 0.29 \\
2 & 0.43 & 0.30 & 0.53 \\
3 & 0.48 & 0.15 & 0.34 \\
\hline
\end{tabular}

ments. No significant differences in the direction expected by the analysis of the RILs were detected (data not shown), making it unlikely that allelic variation at the $E R$ locus led to differences in seed germination. Instead another gene, very closely linked to $E R$ must be responsible for the seed dormancy QTL at this position.

\section{Discussion}

Genetic variation for dormancy in nature has been described mostly in terms of quantitative inheritance (Lane \& Lawrence, 1995). QTL analysis using molecular markers and doubled haploids (DHs) or RILs was used for wheat (Anderson et al., 1993) and barley (Ullrich et al., 1993; Oberthur et al., 1995). In DHs of barley derived from two cultivars with a large difference in seed dormancy, the authors (Ullrich et al., 1993; Oberthur et al., 1995) identified 27 significant loci of which one had by far the largest effect. In Arabidopsis, a comparably large number of loci (14) was identified in a situation where the parental differences were relatively small. However, none of the loci deserved the qualification of a major gene. Nine out of 14 dormancy/germination QTLs were detected in all three germination environments, but the other five were found only under specific germination conditions. Three of 
them acted mainly on germination in the presence of paclobutrazol with a much lower or zero effect when germination was tested in darkness and/or light. The two other QTLs affected germination in darkness and/or light but had practically no effect on germination in the presence of paclobutrazol. The observation of distinct QTLs interacting in different ways with the three germination environments tested confirms the complex relationship between the photocontrol and hormonal control of seed dormancy and germination that has been detected in Arabidopsis through the analysis of mutants (Koornneef \& Karssen, 1994). This QTL $\times$ germination environment interaction suggests, for example, that part of the differences for paclobutrazol resistance in the material are controlled by specific loci that
Fig. 5 The location and effects of the seed dormancy/germination quantitative loci of Arabidopsis. Chromosome numbers are indicated at the top-right corner of each graph, markers are plotted along the abscissa. Selected markers are indicated by + when interaction with environment is still assumed, otherwise by v. Solid curves in the upper part indicate the test statistics (twice the e-log of the likelihood ratio) for the hypothesis of a QTL with no QTL-by-environment interaction assumed. Dashed curves represent the test statistics for the hypothesis of a QTL with QTL-by-environment interaction, which are only shown where this interaction was detected. The overall 5 per cent significance threshold for markers (main effects) was 12.2 and for the marker environment interaction 21.8. Bars along the abscissa indicate two LOD support intervals for the map locations of detected QTLs. In the lower part of each chromosome graph QTL effects (expressed as angular transformed $[\arcsin \sqrt{ }$ ] percentages) are given: $>0$ when Ler alleles have a positive effect on germination and $<0$ when the Col alleles promote germination. In regions where QTL $\times$ environment interaction was significant, the QTL effects are plotted for each germination environment that showed the interaction.
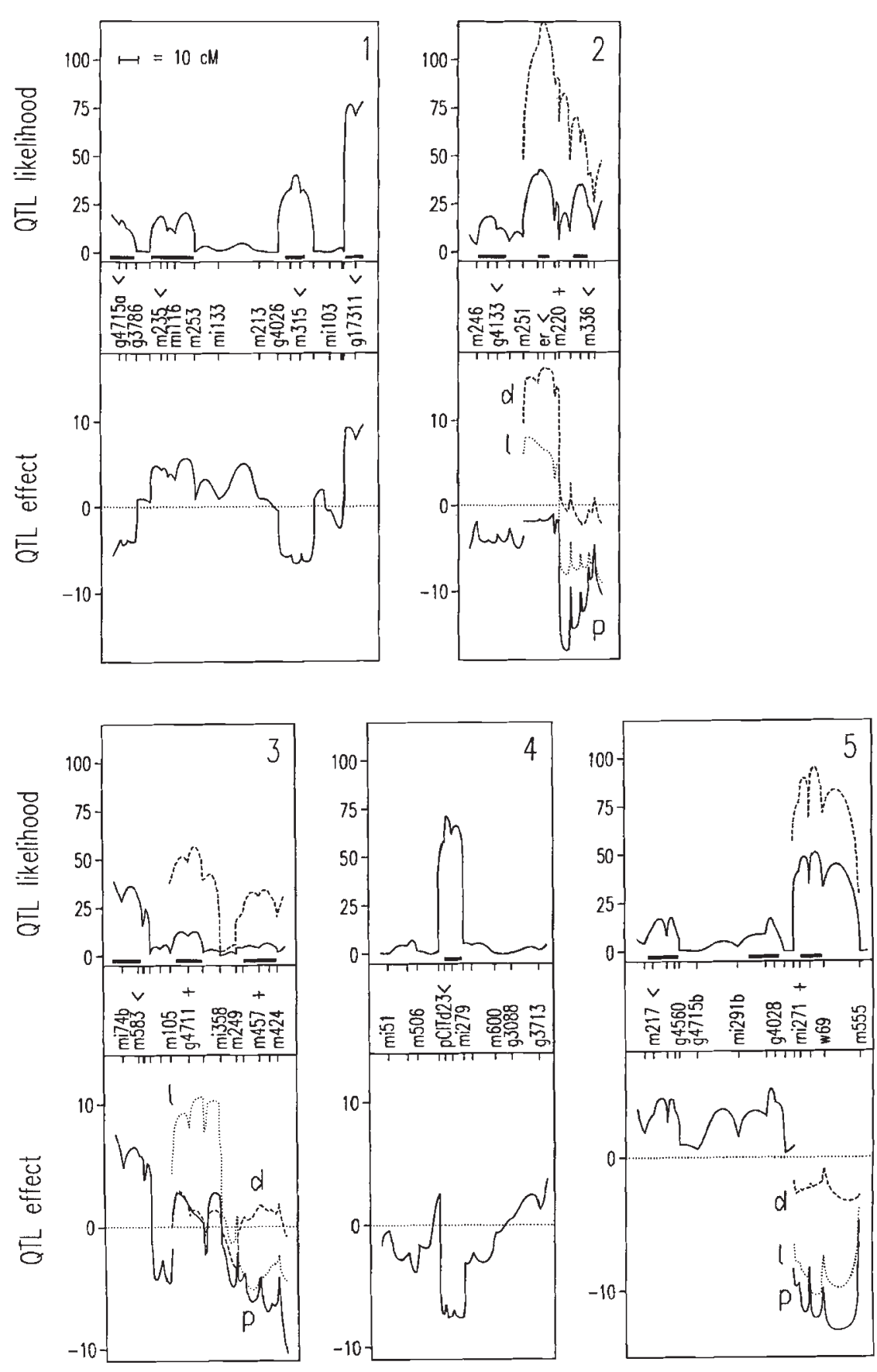
have no effect on germination in darkness. The inhibition of germination by paclobutrazol and other inhibitors of gibberellin (GA) biosynthesis together with the observation that GA-deficient mutants in Arabidopsis do not germinate (Koornneef \& van der Veen, 1980) indicated that GA is required for germination. This GA requirement seems to be related to dormancy through embryonic abscisic acid (ABA) (Koornneef \& Karssen, 1994). However, when other factors (testa components) are relevant this may not lead to differences in GA requirement. In an analysis of two reduced-dormancy mutants (rdol and rdo2) in Arabidopsis, Léon-Kloosterziel et al. (1996a) found that one (rdo2) was more resistant to GA inhibitors than wild type, whereas the other ( $r d o l)$ was not. Apparently, seed dormancy is related to differences in GA requirement for germination in some but not in all situations.

The use of RILs and the application of MQM mapping resulted in improved opportunities to unravel complex genetic traits such as seed dormancy. The mapping of 14 putative QTLs for seed dormancy in Arabidopsis is, together with the location of flowering time genes in the same population (Jansen et al., 1995), one of the first applications combining RILs and MQM mapping. At first sight it might be surprising that such a large number of QTLs were so precisely located in our experiment, as markers explained 8-53 per cent of the total phenotypic variance in the nine environments (Table 3) and correlations between environments were not particularly high (Table 2). A standard interval mapping approach would not detect this number of QTLs, neither in separate analyses per environment nor in an overall analysis based on averages over environments. Separate interval mapping analyses will be more appropriate for QTLs that interact with environment, whereas the analysis based on averages will be more powerful for QTLs that do not interact (because environments then become equivalent to replicates and residual variance is divided by nine). In MQM mapping a joint analysis over all environments and QTLs is performed and interactions between QTLs and environments are now part of the model. For the noninteracting QTLs, only a parameter for main effect is involved; MQM mapping and an overall analysis in interval mapping then exploits the fact that environments become equivalent to replicates, which significantly increases power and precision. For regions displaying putative QTL-environment interactions, main and interaction effects are estimated in MQM mapping, thereby combining the correct estimation of QTL effects over environments of the separate analyses with the advantage of an overall analysis (higher power and precision). Finally, but not less importantly, only in a multiple QTL model as employed by MQM mapping is the genetic variation induced by QTLs located elsewhere on the genome also eliminated, which minimizes the residual error and thereby further increases the power. The two-LOD support intervals for QTL location, as presented by bars in Fig. 5, are in the range of $6-23 \mathrm{cM}$. The relationship between support interval and confidence level has been studied in the simple setting of interval mapping of a single QTL (Van Ooijen, 1992). It has been demonstrated that the confidence level for two LOD intervals can be much higher than 95 per cent for QTLs with major effect, but much lower for QTLs with minor effects. A similar study has not yet been carried out for situations with multiple linked QTLs as encountered in our analysis. Furthermore, the even more complex situation of MQM mapping with QTL-by-environment interaction has not yet been studied. Therefore, at this stage we make no claim about the exact confidence levels of the support intervals presented in Fig. 5.

The data presented in Fig. 4 show considerable genotype-by-environment interaction. The RIL and parental data together suggest epistasis, which is typical of duplicate epistasis. However, because, unlike the RILs, the parents were not randomized, it cannot be excluded that variation within trays influenced the data of the parents in Fig. 4. In MQM mapping, markers and environments are used as cofactors and interactions between markers and environments are also part of the model. Other patterns of interaction, such as two-way interactions between pairs of loci (epistasis), can, in principle, be modelled straightforwardly as well, but we have chosen not to do so because it is hardly possible to study two-way or higher order interactions in a small population of 98 RILs. This is because the number of parameters rapidly exceeds the population size if many QTLs are involved, thereby also reducing the potentials of obtaining significance. Consequently, true interactions which now are not part of the model are contributing to the residual variance. This does not mean that epistasis is absent among the loci that we identified but that it is simply not taken into account in the MQM mapping procedure. In what way ignoring this interaction leads to a different estimate of the size of the QTLs is not known. It is presumed that the position of the QTLs will not change because of epistasis.

In species such as Arabidopsis, maize and barley, where both large numbers of mapped monogenic 
mutants and the position of QTLs are known, it is of interest to see if some of these positions 'colocalize'. In barley, the major seed dormancy QTL coincides with a gene controlling the $\beta$-glucanase activity in the early germination stage, suggesting a biochemical function for the dormancy locus (Han et al., 1995). Candidate mutant loci of Arabidopsis known to be involved in seed/dormancy germination that might overlap with the QTLs found in the present analysis are ABA-related loci such as aba3 located at the top of chromosome 1 (Léon-Kloosterziel et al., 1996b), abi3 and fus3 (near g4711 on chromosome 3; Koornneef, 1994) and abi2 at the bottom of chromosome 5 (R. R. Finkelstein, personal communication), phytochrome loci on the top of chromosome 1 (phyA) and 3 (hy2) and on chromosome 2 (hyl, close to er) and the gibberellin sensitivity locus gai on the top of chromosome 1 (Koornneef, 1994). The coincidence of a QTL locus on chromosome 2 with the $E R$ locus suggested a common locus. However, the comparison of genotypes alone differing for this locus did not indicate an effect of the er mutation on this trait. This implies that 'common' map positions must be judged very carefully and one should realize that it is only rarely possible to test locus identity, because mostly different populations are used to map the mutant locus and the QTL.

The existence of ecotypes that differ much more in germination behaviour than the Ler and Col ecotypes used in the present study (Kugler, 1951; Napp-Zinn, 1975; Ratcliffe, 1976; Alonso Blanco et al., unpublished results) indicates that probably more loci, including some with major gene type effects might be identified. The extensive molecular and genetic analysis of Arabidopsis, when brought together with the analysis of natural variation using approaches as described in this report, should allow the molecular identification and cloning of some of these QTLs, which are of importance both in nature (Evans \& Ratcliffe, 1972) and in crop breeding.

\section{Acknowledgements}

We thank Drs C. Lister and C. Dean for providing the RFLP genotypes for the RILs, the NASC for sending the seeds of the RILs and Dr G. P. Rédei for the LER genotype. The help of Hetty Blankestijn de Vries and Corrie Hanhart with the handling of the plants and the germination tests is greatly acknowledged. We thank Dr Piet Stam for useful discussions. KML-K was supported by grants from the BRIDGE program of the European Union
(BIOT-CT90-0207 and BIOT-CT92-0529) and the Human Frontier Science Program (RG-303/95). C.A.-B. was supported by the Biotechnology TDR program of the EU (grant no. BIO4CT965008).

\section{References}

ANDERSON, J. A., SORRELls, M. E. AND TANKSLEY, S. D. 1993. RFLP analysis of genomic regions associated with resistance to preharvest sprouting in wheat. Crop. Sci., 33, 453-459.

BURR, B. AND BURR, F. A. 1991. Recombinant inbreds for molecular mapping in maize: theoretical and practical considerations. Trends Genet., 7, 55-60.

DERKX, M. P. M. AND KARSSEN, C. M. 1993. Variability in light, gibberellin and nitrate requirement of Arabidopsis thaliana seeds due to harvest time and conditions of dry storage. J. Plant Physiol., 141, 574-582.

EVANS, J. AND RATClifFE, D. 1972. Variation in 'afterripening' of seeds of Arabidopsis thaliana and its ecological significance. Arabid. Inf. Serv. 9, 3-5.

GARBUTt, K. AND WITCOMBE, J. R. 1986. The inheritance of seed dormancy in Sinapis arvensis L. Heredity, 56, $25-31$.

HAN, F., UllRich, S. E., CHIRAT, S., MENTEUR, S., JESTIN, L., SARRAF1, A. ET AL. 1995. Mapping of $\beta$-glucan content and $\beta$-glucanase activity loci in barley grain and malt. Theor. Appl. Genet., 91, 921-927.

JANSEN, R. C. 1994. Controlling the type I and type II errors in mapping quantitative trait loci. Genetics, 138, $871-881$.

JANSEN, R. C. 1996. Complex plant traits: time for polygenic analysis. Trends Plant Sci., 1, 89-94.

JANSEN, R. C. AND STAM, P. 1994. High resolution of quantitative traits into multiple loci via interval mapping. Genetics, 136, 1447-1455.

JANSEN, R. C., VAN OOIJEN, J. W., STAM, P., LISTER, C. AND DEAN, C. 1995. Genotype-by-environment interaction in genetic mapping of multiple quantitative trait loci. Theor. Appl. Genet., 91, 33-37.

KOORNNEEF, M. 1994. Arabidopsis genetics. In: Meyerowitz, E. M. and Somerville, C. R. (eds) Arabidopsis, pp. 89-120. Cold Spring Harbor Laboratory Press, Cold Spring Harbor, NY.

KOORNNEEF, M. AND KARSSEN, C. M. 1994. Seed dormancy and germination. In: Meyerowitz, E. M. and Somerville, C. R. (eds) Arabidopsis, pp. 313-334. Cold Spring Harbor Laboratory Press, Cold Spring Harbor, NY.

KOORNNEEF, M. AND VAN DER VEEN, J. H. 1980. Induction and analysis of gibberellin sensitive mutants in Arabidopsis thaliana (L.) Heynh. Theor. Appl. Genet., 58, 257-263.

KUGLER, 1. 1951. Untersuchungen über das Keimverhalten einiger Rassen von Arabidopsis thaliana (L.) Heynh. Ein Beitrag zum Problem der Lichtkeimung. Beitr. Biol. Pflanzen, 28, 211-243.

LAIBACH, F. 1951. Uber sommer-und winterannuelle 
Rassen von Arabidopsis thaliana (L.) Heynh. Ein Beitrag zur Ätiologie der Blütenbildung. Beitr. Biol. Pflanzen, 28, 173-210.

LANE, M. D. AND LAWRENCE, M. J. 1995. Genetics of seed dormancy in Papaver rhoeas. Heredity, 75, 84-91.

LÉON-KLOOSTERZIEL, K. M., VAN DER BUNT, G., ZEEVAART, J. A. D. AND KOORNNEEF, M. 1996a. Arabidopsis mutants with a reduced seed dormancy. Pl. Physiol., 110, 233-240.

LÉON-KLOOSTERZiel, K. M., AlvAReZ-Gil, M., RUiJs, G. D., JACOBSEN, S. E., OLSZEWSKI, N. E., SCHWARTZ, S. H. ET AL. 1996b. Isolation and characterization of abscisic aciddeficient Arabidopsis mutants at two new loci. Plant J., 10, 655-661.

LISTER, C. AND DEAN, D. 1993. Recombinant inbred lines for mapping RFLP and phenotypic markers in Arabidopsis thaliana. Plant J., 4, 745-750.

MEYerowitz, E. M. 1987. Arabidopsis thaliana. Ann. Rev. Genet, 21, 93-111.

NAPP-ZINN, K. 1975. On the genetical basis of light requirement in seed germination of Arabidopsis. Arabid. Inf. Serv. 12, 10.

OBERTHUR, L. E., DYER, W., ULLRICH, S. E. AND BLAKE, T. K.
1995. Genetic analysis of seed dormancy in barley (Hordeum vulgare L.) J. Quant. Trait Loci [on line]. Available on World Wide Web: http://probe.nalusda. gov:8000/otherdocs/jgtl1995-05/dormancy.html.jqtl

PlatenkAMP, G. A. J. AND SHAw, R. G. 1993. Environmental and genetical maternal effects on seed characters in Nemophila menziess. Evolution, 47, 540-555.

RATCLIFFE, D. 1976. Germination characteristics and their inter- and intra-population variability in Arabidopsis. Arabid. Inf. Serv., 13, 34-45.

RÉUEI, G. P. 1962. Single locus heterosis. Z. Vererbl, 93, $164-170$.

simpson, G. M. 1990. Seed Dormancy in Grasses. Cambridge University Press, Cambridge, UK.

ULLRICH, S. E., HAYES, P. M., DYER, W. E., BLAKE, T, K. AND CLANCY, J. A. 1993. Quantitative trait locus analysis of seed dormancy in 'Steptoe' barley. In: WalkerSimmons, M. K. and Ried, J. L. (eds) Pre-harvest Sprouting in Cereals 1992, pp. 136-145. Am. Assoc. Cereal Chem. St Paul, MN.

VAN OOIJEN, J. W. 1992. Accuracy of mapping quantitative trait loci in autogamous species. Theor. Appl. Genet., 84, 803-811. 